\title{
Simulation of an Asynchronous Machine by using a Pseudo Bond Graph
}

\author{
Gregorio Romero $^{\mathrm{a}}$, Jesus Felez ${ }^{\mathrm{a}}$, Joaquin Maroto ${ }^{\mathrm{a}}$, M. Luisa Martinez ${ }^{\mathrm{a}}$ \\ ${ }^{a}$ ETS Ingenieros Industriales, Univ. Politécnica de Madrid, Jose Gutierrez Abascal, 2, 28006 Madrid, Spain. \\ Email: \{gregorio.romero, jesus.felez, joaquin.maroto, luisa.mtzmuneta\}@upm.es
}

\begin{abstract}
For engineers, computer simulation, is a basic tool since it enables them to understand how systems work without actually needing to see them. They can learn how they work in different circumstances and optimize their design with considerably less cost in terms of time and money than if they had to carry out tests on a physical system. However, if computer simulation is to be reliable it is essential for the simulation model to be validated. There is a wide range of commercial brands on the market offering products for electrical domain simulation (SPICE, LabVIEW PSCAD,Dymola, Simulink, Simplorer,...). These are powerful tools, but require the engineer to have a perfect knowledge of the electrical field. This paper shows an alternative methodology to can simulate an asynchronous machine using the multidomain Bond Graph technique and apply it in any program that permit the simulation of models based in this technique; no extraordinary knowledge of this technique and electric field are required to understand the process .
\end{abstract}

Keywords: Bond Graph, pseudo Bond Graph, simulation, asynchronous machine, electric. PACS: 07.05.Tp Computer modeling and simulation, 84.50. $+\mathrm{d}$ Electric motors.

\section{INTRODUCTION}

In electrical engineering, simulation is an indispensable tool when working with complex systems. Studying electrical systems is relatively simple if their behaviour is being analysed under a permanent regime and they consist of few nodes. However, when the systems are complex and require a qualitative and quantitative analysis of the transitory regimes [7], the problems also become considerably more complex, up to a point that traditional modelling and calculation tools are clearly unsuited to the purpose.

The importance of asynchronous motors based on the concept of a revolving magnetic field put forward by Ferraris and Tesla, can be put down to its simple robust construction, particularly in the case of the squirrel-cage rotor, which makes them work in the most adverse circumstances, furnishing excellent service with minimum maintenance. Nowadays it can be said that $80 \%$ of industrial electrical motors use this machine. The most serious drawback arises out of the difficulty in regulating their speed; hence in electrical attraction they give way to direct current motors which are more suited to this service. Recently, however, with the development of electronic devices such as inverters and cycle-converters which allow a variable frequency to be obtained from the constant mains frequency, together with the introduction of microprocessors in power electronics, great changes are taking place; asynchronous motor applications in variable speed electrical operation are beginning to become more extensive.

When it comes to studying the model associated with an asynchronous machine, it can either be approached from the equivalent circuit or after applying the special vectors theory, which will be explained in the following sections.

\section{EQUIVALENT IN-CIRCUIT ASYNCHRONOUS MOTOR MODEL}

As with direct current transformers and motors, the purpose of an equivalent circuit of an asynchronous motor is to obtain a power supply that will explain the behaviour of the machine [3], wich require to have a perfect knowledge of the electrical field, such as we can read in the next two parragraphs of this chapter. 
The induction machine comprises a stator and a rotor [8]. The inducer is usually placed in the stator, powered by a three-phase supply. It is made up of stacked plates and a winding comprising three $2 \cdot p$ pole coils $120^{\circ}$ out of phase in space. When three-phase currents of frequency $f_{l}$ are made to pass through them a rotating wave is produced with a magnet motor force (m.m.f.) that is distributed sinusoidally along the periphery of the air-gap as a revolving flux whose speed can be expressed by the speed of synchronism $n_{l}$. In this way, the rotor is induced and the currents flowing through it appear as a result of the interaction with the stator flux. As the current flows through the rotorconductors a force appears in them whose value is obtained by applying Laplace's Law.In this type of machine the motor must rotate at a speed lower than that of synchronism $\left(n<n_{1}\right)$, it being an asynchronous regime speed, which can be known by letting $s$ be the sliding to give the quotient between the difference in speeds $n$ and $n l$, and the speed of synchronism. If it is wished to obtain the electrical behaviour equation of the stator and rotor, it must be borne in mind that the coils have resistances of $R_{1}$ y $R_{2}\left[{ }^{\Omega /}\right.$ fase $]$ and, in addition there are dispersion fluxes in the stator and rotor windings which lead to the self-inductions $L_{d 1}$ y $L_{d 2}$.

Taking account of the relation between the rotor and stator frequencies and the losses in the magnetic nucleus, after reduce the rotor to the stator is possible to obtain the equivalent circuit diagram [13].

The bond graph method was invented by Henry Paynter in 1959 and carried to application by Karnopp and Rosenberg [2][4]. This technique was developed as an appropriate tool for modeling all types of systems. It is based on the topologic representation of different mechanisms regarding exchange, storage and energy dissipation in any dynamical system. In its methodology, energy is represented through two kinds of general variables: the power variables (effort and flow) and energy variables (momentum and displacement). The power variables are related to energy variables through integral relationships.

Once the equivalent circuit of an asynchronous motor has been generated, the Bond Graph diagram is obtained directly substituting the resistances and circuit coils by $R$ and $I$ elements with a 1 junction (same flow or intensity) and the parallel branches for type 0 junction (same effort or voltage) [6], such as we can see in the figure 1.

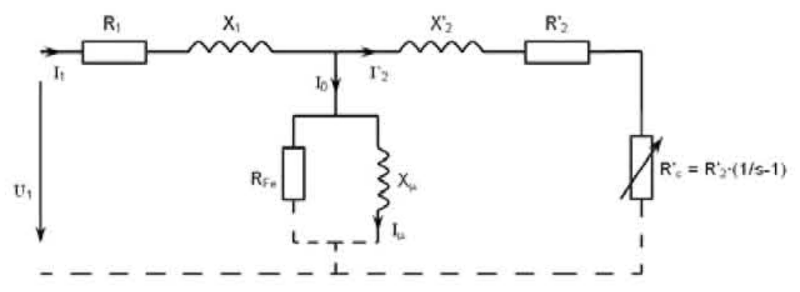

(a)

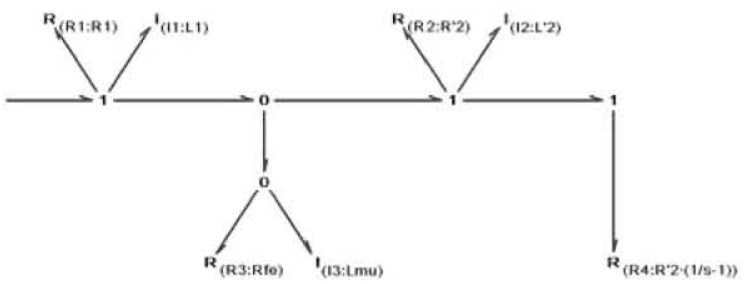

(b)

FIGURE 1. Equivalent circuit. (a) Diagram, (b) Bond Graph.

In the previous bond graph model the identification of each element (name and value) are writen between parenthesis down the element (ie, the value associated to the resistance with the name " $R 3$ " is "Rfe").

The speed of synchronism is constant and is imposed by the mains frequency, which means there is no problem. However, the shaft speed varies depending on the machine's dynamic behaviour. In the above Bond Graph model (figure 1.b) there is no variable to directly represent the speed, which means it must be obtained indirectly. Taking account the differential equation of the shaft $(M=J \cdot d \Omega / d t \rightarrow d \Omega / d t=M / J)$, a supplementary diagram is produced where the speed variable $\Omega$ appears (speed of the Inertance 14 ), which will be required to define the load resistance $R_{c}$. of the equivalent circuit Bond Graph model of the figure 1.b.

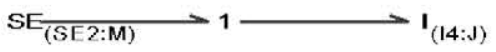

FIGURE 2. Supplementary Bond Graph of an asynchronous machine

In the supplementary Bond Graph of the previous figure the effort source (SE2) represents the torque applied to the shaft and the inertia port (I4) represents the moment of inertia. The effort source is dependent on the flow from the inertia port $I 2$ (current $I_{2}$ - figure 1.b.) and on the flow from inertia port $I 4$ (speed $\Omega$-figure 2).

Thus, by substituting the values of the parameters in the preceding paragraph and noting "flow of a -Inertance-" like "_ $V$ " plus 'name of the Inertance' plus "_", the following expressions for $M \mathrm{y} R_{c}$ are obtained directly: 


$$
\begin{aligned}
M & =\frac{3 \cdot{ }_{-} V I 2_{-}{ }^{2} \cdot R^{\prime} 2}{2 \cdot \pi \cdot \frac{f_{1}}{p}-{ }_{-} V I 4} \\
R_{c} & =\frac{-V I 4_{-} \cdot R^{\prime 2}}{2 \cdot \pi \cdot \frac{f_{1}}{p}-{ }_{-} V I 4}
\end{aligned}
$$

Thus, the end model in a Bond Graph would be as in the figure below:

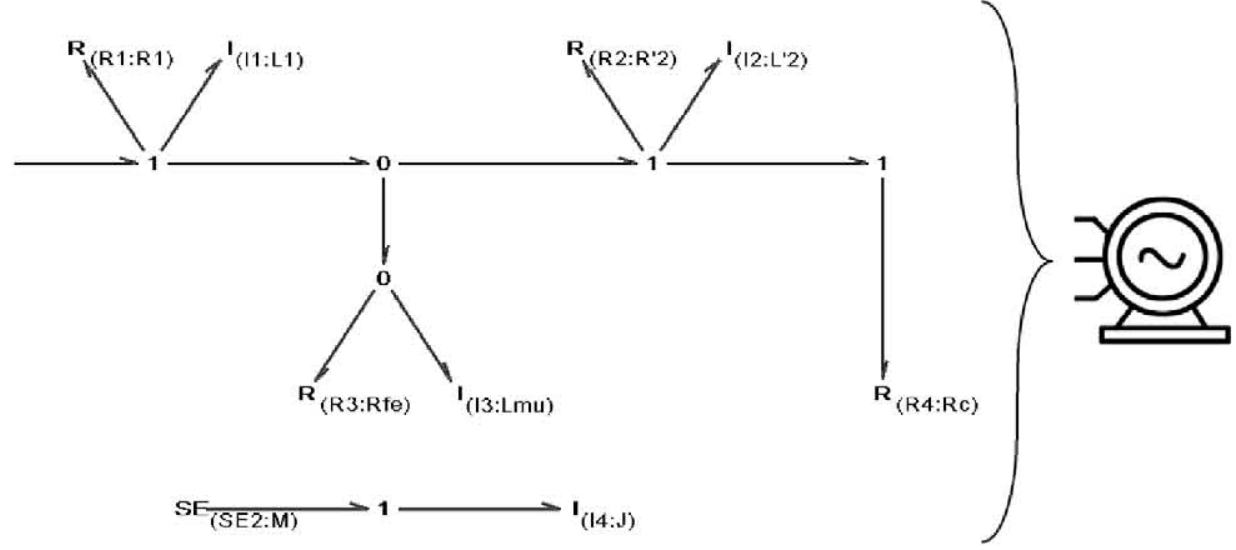

FIGURE 3. Asynchronous machine single phase model using a Bond Graph

This model is very simple and useful to simulate permanent regimes, but when the systems require a qualitative and quantitative analysis of the transitory regimes, we need a more complex model to the purpose, such as a asynchronous motors based on the concept of a revolving magnetic field.

If we analyze a asynchronous motor model in spatial vectors, which requires knowledge in electric field, we can obtain a equivalent circuit diagram and we could obtain the equivalent model in Bond Graph directly from it such as in the previous figure [11][12]. The objetive of the next chapter is show to the readers other alternative obtaining a Bond Graph model directly from the equations system, not from the equivalent circuit diagram.

\section{ASYNCHRONOUS MOTOR MODEL IN SPATIAL VECTORS}

Another more modern and powerful way of studying an asynchronous machine is by the theory of spatial vectors [9]. In an asynchronous machine there is a set of magnitudes that evolve sinusoidally in space. In order to represent a sinusoid in a dual pole machine, it is sufficient to indicate its maximum value and the position in space where this maximum value is obtained. This may be done by using a spatial vector whose length or modulus is proportional to the maximum value of the sinusoid and whose position or argument coincides with the point in space where the positive maximum of the sinusoid is attained. In machines with more than two poles, the magnitudes have more than one positive maximum. For their spatial vector to be unique, electrical angles are worked with. Hence the expression, spatial favours, which is more appropriate than spatial vectors.

$$
\vec{a}(t)=A(t) \cdot e^{j \omega t}
$$

The mathematical transformation usually used to define the spatial vectors corresponding to each of the variables in a three-phase system $\left(x_{1}(t), x_{2}(t), x_{3}(t)\right)$ is: 


$$
\vec{x}(t)=\left[\begin{array}{lll}
1 & e^{j \frac{2 \pi}{3}} & e^{j \frac{4 \pi}{3}}
\end{array}\right] \cdot\left[\begin{array}{l}
x_{1}(t) \\
x_{2}(t) \\
x_{3}(t)
\end{array}\right]
$$

It, therefore, does not correspond with the variable of any of the three phases, but is made up of the sum of all three. Moreover, it is possible to take as a reference system either one linked to the stator or one linked to the rotor.

In order to develop a model of this type of motor, we first need the equations controlling the workings of the asynchronous machine under a dynamic regime (magnetic field, stator and rotor equations, torque developed by the machine and the dynamic equation of the mechanical system) and assume the machine stator and rotor supply voltage and load torque are known. By selecting a reference system whose direct axis coincides with the magnetising field of the rotor, the equations can be simplified.

The following simplified hypotheses are accepted:

1. The motor has a three-phase stator-like winding, which, if connected by a star connection, will have an insulated neutral pin.

2. The spatial harmonics of the m.m.f. and the air-gap induction are aroused.

3. The stator and rotor iron nucleus have infinite permeability; that is, they cannot be saturated.

4. The film effect of the conductors and the losses in the iron are negligible.

5. The machine air-gap is constant and of negligible thickness. The shaving effects can also be ignored.

Taking the three phase voltage and naming the components of each vector with the sub-indices ' $d$ ' and ' $q$ ' according to the direct axis and the quadrature axis respectively, the voltage source powered model can be written in the following form:

$$
\begin{gathered}
\frac{u_{s d}}{R_{s}}=i_{s d}+T_{s} \cdot \sigma \cdot \frac{d i_{s d}}{d t}-\omega_{m r} \cdot T_{s} \cdot \sigma \cdot i_{s q}+T_{s} \cdot(1-\sigma) \cdot \frac{d i_{m r}}{d t} \\
\frac{u_{s q}}{R_{s}}=i_{s q}+T_{s} \cdot \sigma \cdot \frac{d i_{s q}}{d t}+\omega_{m r} \cdot T_{s} \cdot \sigma \cdot i_{s d}+\omega_{m r} \cdot T_{s} \cdot(1-\sigma) \cdot i_{m r} \\
i_{s d}=i_{m r}+T_{r} \cdot \frac{d i_{m r}}{d t} \\
i_{s q}=T_{r} \cdot i_{m r} \cdot\left(\omega_{m r}-\omega_{e}\right) \\
M_{e}=k \cdot i_{s q} \cdot i_{m r} \\
M_{e}-M_{c}=\frac{J}{p} \cdot \frac{d \omega_{e}}{d t}
\end{gathered}
$$

This system of equations of the asynchronous machine does not have any equivalent circuit and does not provide any intuitive understanding of the physical reality. Due to this fact, transferring it to a Bond Graph is done differently from the equivalent in-circuit described in the previous chapter. On this occasion a diagram is drawn to generate the system of equations, getting away from the idea that that every element of the diagram represents a physical phenomenon [1].

The application of Bond Graph to some domains such as chemical or thermal processes is not straight forward since this kind of system is normally described using energy input, output and accumulation of different forms. For this reason, Pseudo-Bond Graphs are employed, in which the definition of the variables is not exactly the same as in real Bond Graphs [4] (in Bond Graph models the effort of a bond multiplied by he flow of the same bond is equal to the power that are transmited by this bond from a part of the model to another one). The Pseudo-Bond Graphs provides more flexibility when using coupled differential equations, without losing the advantages of Bond Graph methods. One of the disadvantages of Pseudo-Bond Graphs is that they cannot be readily coupled to other energy domains as true Pseudo-Bond Graphs do, except by means of some ad hoc elements that do not obey the rules of normal Bond Graph elements. The previous equations system is difficult to be represented using true Bond Graphs [14] but can be treated with Pseudo-Bond Graphs in a natural way. So, we shall test another recourse offered by the technique approached in this paper, but differently from the approach in the equivalent in-circuit. 
In order to approach the system of equations in a Pseudo-Bond Graph, flow sources and inertia ports will be used with each addend being introduced as the flow from a flow source, and to join the terms type ' $\theta$ ' nodes will be used where the flows are added together. This has been done differently from the usual way in order to be able to operate both with the flow and the Inertia ports, as well as with the integral and the derivative of the flow. By proceeding in this way, the equations will gradually appear in the flows on the bond regardless of the size of each variable or each addendum.

Firstly, it is essential to perform the conversion from the voltage inputs $u_{1}, u_{2}$ and $u_{3}$ to the stator voltage referred to the fixed stator reference system $\left(u_{s a}, u_{s b}\right)$.

$$
\begin{gathered}
u_{s a}=\frac{3}{2} \cdot u_{1} \\
u_{s b}=\frac{\sqrt{3}}{2} \cdot\left(u_{2}-u_{3}\right)=\frac{\sqrt{3}}{2} \cdot u_{2}-\frac{\sqrt{3}}{2} \cdot u_{3}
\end{gathered}
$$

The first equation can be directly represented and since the second equation comprises two addenda, each one must be introduced as a flow source with both then being added in a type ' 0 ' node, where the output bond flow is the sum of input flows.

$$
\mathrm{Sr} \underset{(\mathrm{SF} 1:(3 / 2) \cdot \mathrm{u} 1)}{\longrightarrow}{ }_{(\mathrm{usa}) 1)}
$$

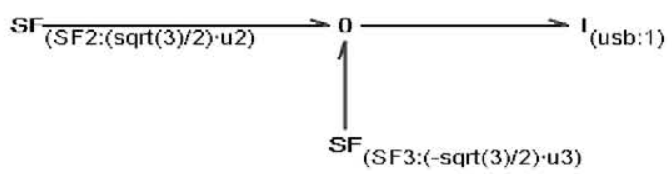

FIGURE 4. Pseudo Bond Graph corresponding voltage of the stator referred to the fixed stator reference system

To be able to refer to the different variables in successive equations and be able to see them in the results output, " $u s a$ " and "usb" type Inertia ports have been introduced, both with a unit value. So, the variables associated with the "usa" port (flow in electric domain or speed in mechanic) and its time integral will be written as "Vusa " and "Xusa_" respectively. Subsequently, it is necessary to change over to $d q$ coordinates with the following result:

$$
\begin{gathered}
u_{s d}=\cdot u_{s a} \cos \left(\varphi_{r m}\right)+u_{s b} \cdot \operatorname{sen}\left(\varphi_{r m}\right) \\
u_{s q}=-u_{s a} \cdot \operatorname{sen}\left(\varphi_{r m}\right)+u_{s b} \cdot \cos \left(\varphi_{r m}\right)
\end{gathered}
$$

In these equations, since reference is made to the variables $u_{s a}$ and $u_{s b}$ obtained in the pseudo Bond Graph in figure 4, we refer to them as "Vusa_" and "Vusb_". The variable $\phi_{r m}$ will appear later and has been denominated as "phi" in the next figure.
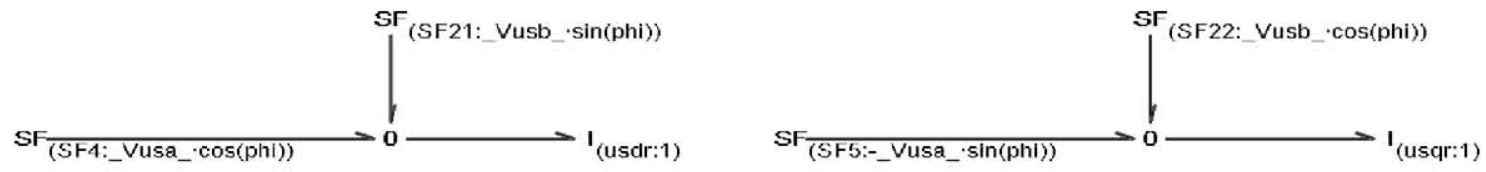

FIGURE 5. Pseudo Bond Graph corresponding to the changeover to $d q$ coordinates

After solving $\frac{d i_{s q}}{d t}$ y $\frac{d i_{s d}}{d t}$ of the machine equations (5) and (6), the following expressions are obtained:

$$
\begin{gathered}
\frac{d i_{s q}}{d t}=\frac{u_{s q}}{T_{s} \cdot \sigma \cdot R_{s}}-\frac{i_{s q}}{T_{s} \cdot \sigma}-\omega_{m r} \cdot i_{s d}-\omega_{m r} \cdot \frac{(1-\sigma)}{\sigma} \cdot i_{m r} \\
\frac{d i_{s d}}{d t}=\frac{u_{s d}}{T_{s} \cdot \sigma \cdot R_{s}}-\frac{i_{s d}}{T_{s} \cdot \sigma}+\omega_{m r} \cdot i_{s q}-\frac{(1-\sigma)}{\sigma} \cdot \frac{d i_{m r}}{d t}
\end{gathered}
$$


In these equations the variables $u_{s q}$ y $u_{s d}$ correspond to the variables "_Vusdr_" and "_Vusqr " in figure 5 respectively. On this occasion, in order to be able to refer to the integrals of the variables $i_{s q}$ and $i_{s d}$, the notations "Xdisq" and "Xdisd" will be used respectively. Also, the variable $\omega_{m r}$ will be obtained in a subsequent equation and will be referred to as "omemr" in the next figure.

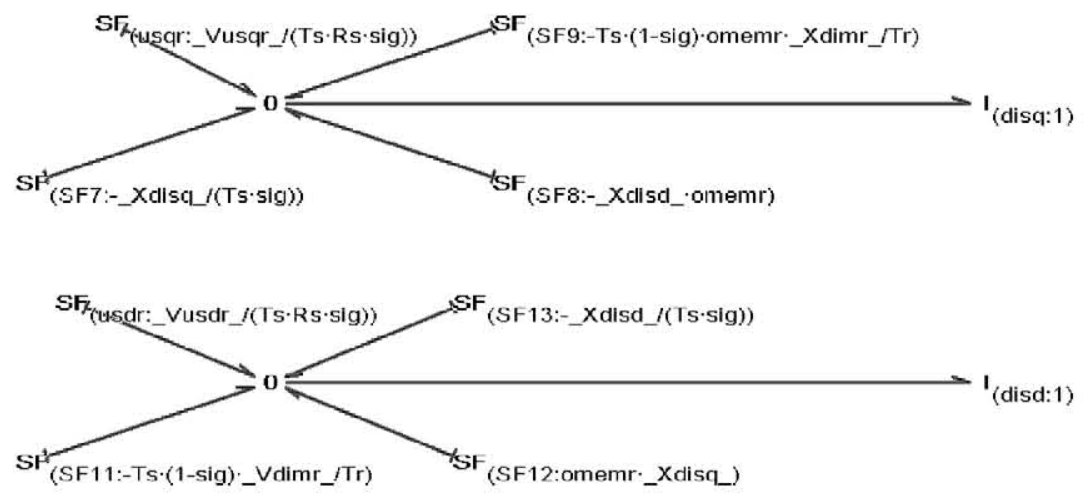

FIGURE 6. Pseudo Bond Graph corresponding to the machine equations

Regarding the rotor magnetization current $i_{m r}$ corresponding to the equation (7), it is possible to formulate it by its derivative.

$$
\frac{d i_{m r}}{d t}=\frac{i_{s d}}{T_{r}}-\frac{i_{m r}}{T_{r}}
$$

In the above expression the variable $i_{s d}$ corresponds to the inertia port flow integral "disd" in figure 6 , it being represented as "Xdisd ". The integral corresponding with the port introduced to represent the equation above these lines will be denoted as "Xdimr".

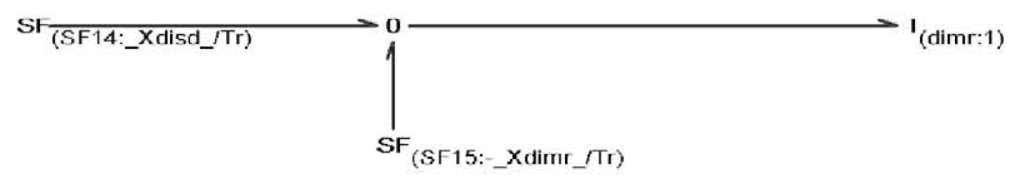

FIGURE 7. Pseudo Bond Graph corresponding to the obtaining of $i_{m r}$

The value of the electrical velocity $\omega_{e}$, can be found from the dynamic torque equation (10) and substituting the value of the torque developed by the machine $M_{e}$ for the value described in (9):

$$
\frac{d \omega_{e}}{d t}=\frac{p}{J} \cdot k \cdot i_{s q} \cdot i_{m r}-\frac{p}{J} \cdot M_{c}
$$

where all the existing variables are known and where the load torque $M_{c}$ is an input parameter.

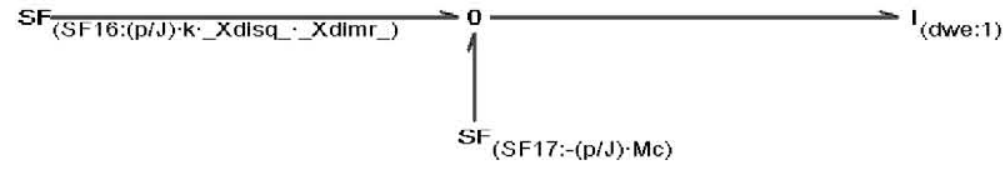

FIGURE 8. Pseudo Bond Graph corresponding to the obtaining of $\omega_{e}$

Finally, after finding the value of the variable $\omega_{m r}$ from equation (8) the following expression is obtained 


$$
\omega_{m r}=\frac{i_{s q}}{T_{r} \cdot i_{m r}}+\omega_{e}
$$

To formulate this expression in a pseudo Bond Graph it is sufficient to introduce both addenda, just as was done in equations (11) to (14) in figures 4 and 5.

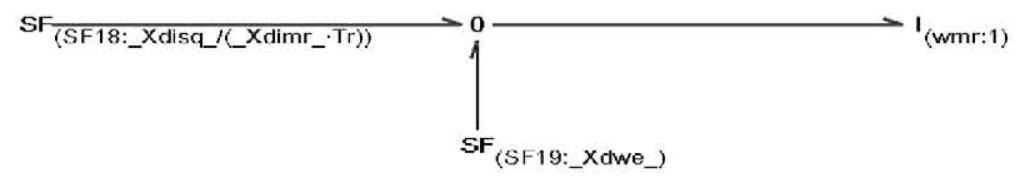

FIGURE 9. Pseudo Bond Graph corresponding to the obtaining of $\omega_{m r}$

The variables $\omega_{m r} \mathbf{y} \phi_{m r}$ in figures 5 and 6 had been pending their appearance in the final equations. The first variable was called "omemr" and now corresponds with "Vwmr_", while the second is the integral of the first, whose provisional name was "phi" and must be changed for "Xwmr_". Making these ultimate changes, the pseudo Bond Graph corresponding to the asynchronous machine in spatial vectors is obtained, as shown in the following figure:
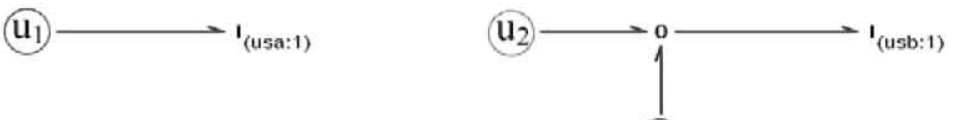

(u)
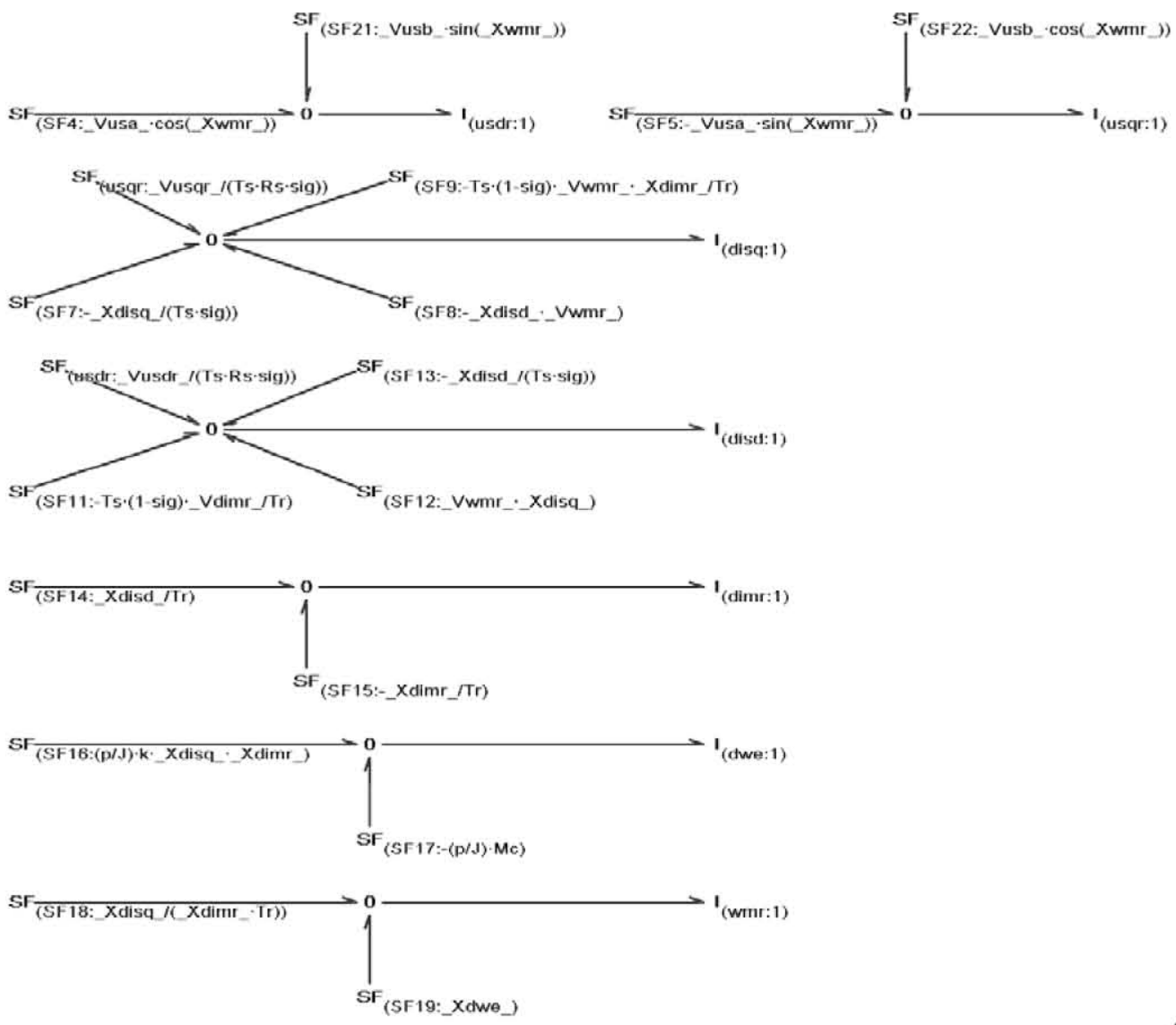

FIGURE 10. PseudoBond Graph corresponding to an asynchronous machine in spatial vectors. 
In order to validate the developed model, we shall now proceed to simulate in a new version of the software $B o n d y n^{\circ}[5]$ the direct start-up of an asynchronous machine connecting it with a star connection directly to a threephase power supply in the following chapter. We proceeded to make the same model in Simulink ${ }^{\circ}$ using a block diagram, which means applying the Laplace transformation to the asynchronous machine model equations, to validate both simulations.

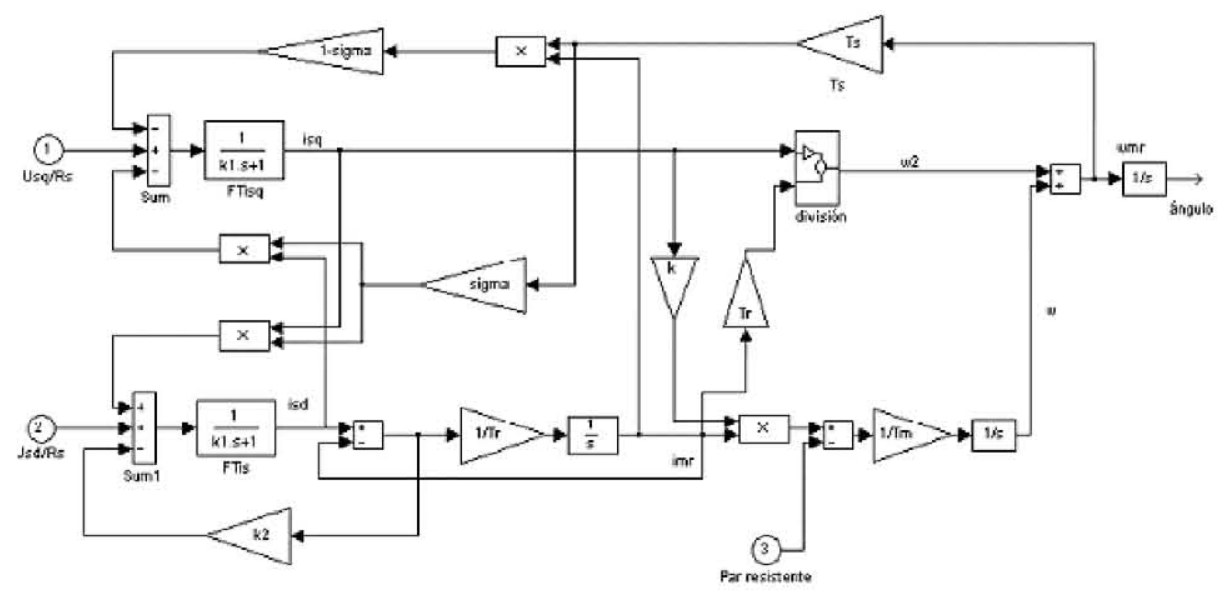

FIGURE 11. Asynchronous machine block diagram in spatial vectors in Simulink ${ }^{\odot}$

\section{DIRECT START-UP OF AN ASYNCHRONOUS MACHINE}

This start-up method is the simplest one and is used in small power motors and consists in starting the motor by connecting it to its designated voltage. This device lets motors be started even under full load, if the power supply admits the current peak at the instant of start-up.

In the Bond Graph technique, the effort sources (voltage) are ideal sources and generate a constant potential difference between the terminals and regardless of the load. Unlike ideal sources, the potential difference produced by real sources, are dependent on the load to which they are connected.

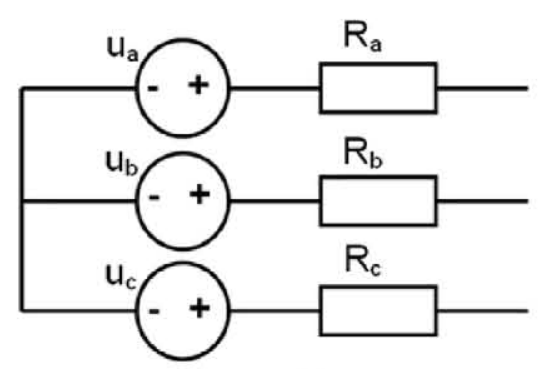

(a)

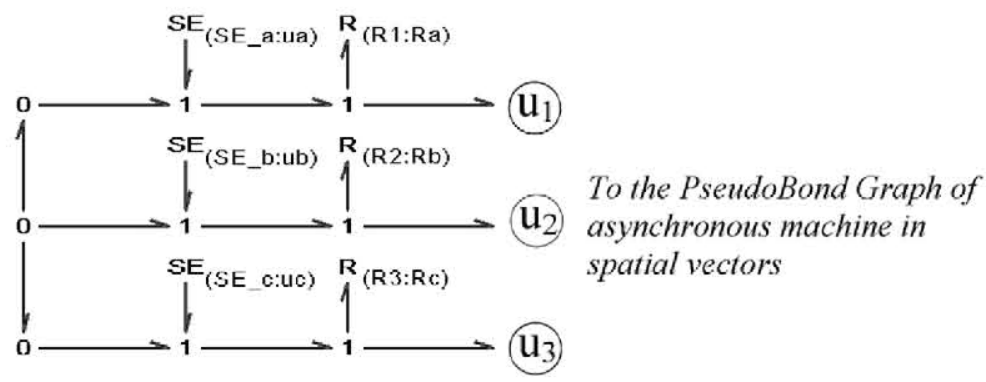

(b)

FIGURE 12. Three-phase voltage source. (a) Diagram, (b) Bond Graph.

A real voltage source may be considered an ideal voltage source, $U$, in series with a internal resistance $R_{g}$, (fig. 12). In order to obtain real sources in a Bond Graph, a resistance port needs to be added to act as $R_{g}$ using a node 1 , so that it will be in series. By joining the single-phase effort forces in an appropriate star or triangular layout, threephase systems can be attained. In the case under study, three-phase, star voltage sources can be obtained by connecting the different effort sources in type $I$ nodes, and subsequently, all of them in a type 0 node.

Once the pseudo Bond Graph model simulations set out here in Bondyn ${ }^{\circ}$ and the one in Simulink ${ }^{\circ}[15]$ have been performed the results obtained can be checked and validated using the following figures.

Firstly, in both cases it can be seen how the torque is at its when the motor reaches $80 \%$ of its speed; at this instant, the peak of intensity is considerably dampened. 


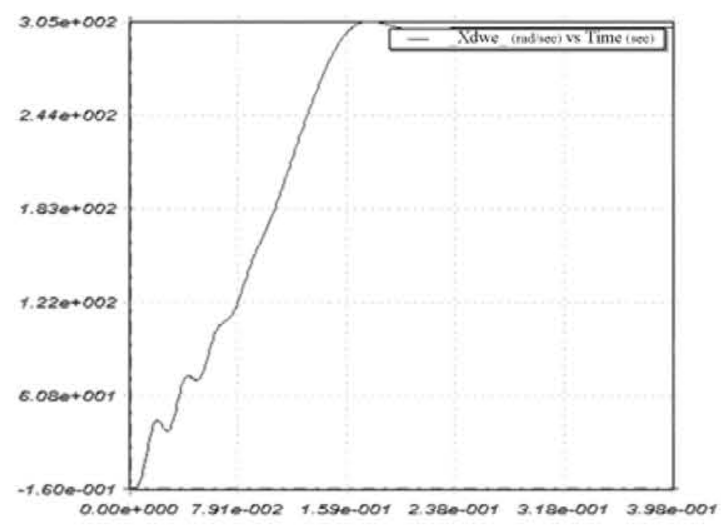

(a)

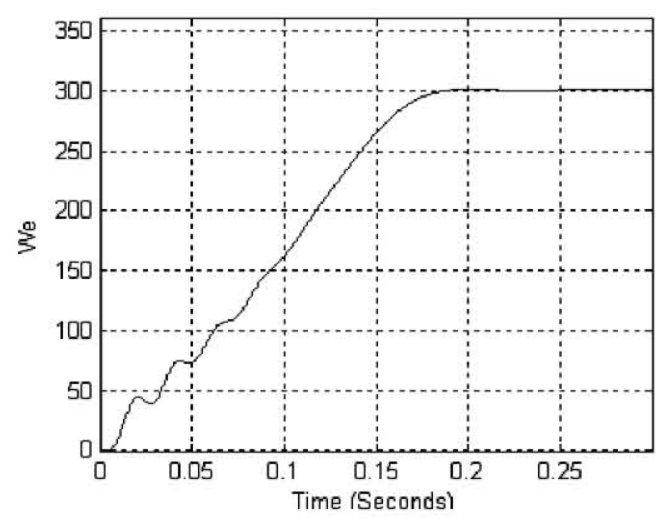

(b)

FIGURE 13. Rotor angular velocity $\omega_{e}$. (a) Bondyn ${ }^{\circ}$, (b) Simulink ${ }^{\circ}$.

By performing both simulations, it can also be seen how the peak of intensity on connection is very high; around four to eight times the nominal intensity. The torque during start-up is always greater than the nominal torque, particularly for complex modern cage motors, for which reason this procedure is not recommended if the start-up needs to be done slowly and progressively.

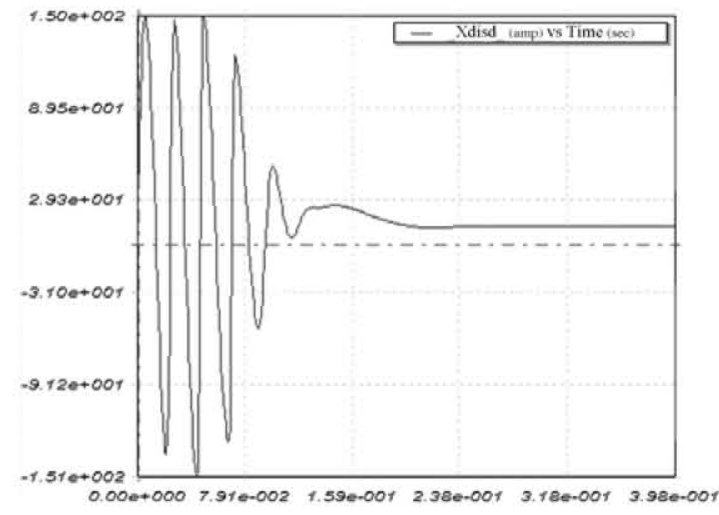

(a)

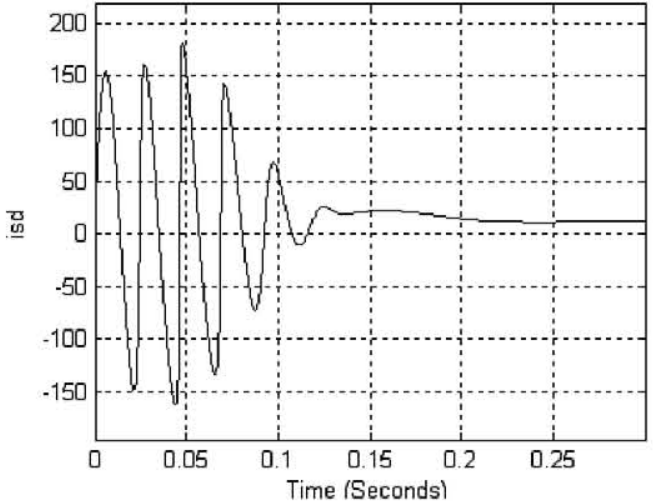

(b)

FIGURE 14. Intensity $I_{s d \text {. (a) Bondyn }}{ }^{\circ}$, (b) Simulink ${ }^{\circ}$.

After examining the different graphs obtained from both simulations, it is clear that the same results are obtained from the asynchronous machine with the model developed here and with Simulink ${ }^{(\theta)}$ after applying the Laplace transformation to the asynchronous machine model equations.

The advantage of the model here developed is that anybody who are simulating anything in a Bond Graph software and want assume a model like the model here shown to connect to the rest of the model don't need simulate it in other tool; only it's necessary transform the equations system to a a Bond Graph or Pseudo Bond Graph model by using the different topology of the nodes and ports.

\section{CONCLUSIONS}

For engineers, simulation and especially computer simulation, is a basic tool since it enables them to understand how systems work without actually needing to see them. They can learn how they work in different circumstances and optimize their design with considerably less cost in terms of time and money than if they had to carry out tests on a physical system. However, if computer simulation is to be reliable it is essential for the simulation model to be validated.

It may be said that the Bond Graph technique is a simple and effective mathematical modelling technique that, if it's possible, lets the model be understood without losing the physical sense of each of its components [10]. 
As an ultimate conclusion, if starting out from a mathematical model that does not provide an intuitive understanding of the physical reality, as is the case when studying asynchronous motors with the theory of spatial vectors that we have dealt with, the model may also be approached by means of a Pseudo Bond Graph.

\section{ACKNOWLEDGMENTS}

This work belongs to project DPI2002-2198 and has been developed under the support of the Spanish

Administration in the programs of the Ministry of Science and Technology related to Research in Transportation and Design and Industrial Production.

\section{REFERENCES}

1. D. Sahm. A 2-axis, bond graph model of the dynamics of synchronous electrical machines. Journal Franklin Inst., Vol. 308/3 , Sept. , pp. 205-218. 1979.

2. R. Rosenberg, D. Karnopp, Introduction to Physical Dynamics Systems., McGraw-Hill Book Company, New York 1983.

3. K. Sirivadhna, et al. The application of Bond Graphs to electrical machines and power engineering. IEEE Tr. on Power Applications and Systems, Vol. PAS-102, No. 5, pp. 1170-11782. 1983.

4. D. Karnopp, D. Margolis, R. Rosenberg, System Dynamics: A Unified Approach., John Wiley \& Son Inc., New York 1990.

5. J. Felez, C. Vera, R. Cacho. BONDYN: A Bond Graph based simulation program Trans. of ASME, J. Dyn. Syst. Measurement and Control, Vol. 112, No.3, pp.717-727. 1990.

6. D. Karnopp. State Functions and Bond Graph Model for Rotary Multi-Winding Electrical Machines. J. Franklin Inst., 328/1, pp. 43-58. 1991.

7. P. K. Kovács. Transient Phenomena in Electrical Machines, Elsevier Sc.Publishers, Amsterdam. 1994.

8. P.C. Krause, O. Wasynczuk, S. Sudhoff. Analysis of Electric Machinery. IEEE Press, N. Y. 1995.

9. D.W.Novotny, T.A. Lipo. Vector control and dynamics of AC drives. $1^{\text {st }}$ ed. Oxford: Clarendon Press. 1996.

10. W. Borutzky, F. Cellier. Tearing in bond graphs with dependent storage elements, Proceedings CESA'96 Symposium on Modelling, Analysis and Simulation, Lille, France, vol.2, pp.1113-1119, 1996.

11. S. Junco, D. Marelli, M. Romero.. Bond Graph Modelling and Simulation of Electrical Machines. Proceedings Int'1. Conf. MSO'97 (Singapore, Aug. 11-13), IASTED/Acta Press, Calgary, Canada1997.

12. S. Junco. Modelizacion y analisis no lineal de la maquina de induccion mediante bond graphs vectoriales de potencia real y compleja. Revista Electrónica de la Facultad de Ciencias Exactas, Ingeniería y Agrimensura. Universidad Nacional de Rosario. Vol. I, No III. 1999.

13. D. Gaude, J.P. Masson, M.A. Raulet, M. Darces. Modelling of an asynchronous single phase motor. Electrimacs 99 : modelling and simulation of electric machines converters and systems : Lisbao, 14-16 September 1999.

14. J. Kim, M. D. Bryant. Bond Graph model of a squirrel cage induction motor with direct physical correspondence. Journal of Dynamic Systems, Measurement, and Control, Vol. 122, Issue 3, pp. 461-469. 2000.

15. J.H. Mathews, F. Kurtis, K. Fink, Numerical Methods Using MatLab. Prentice Hall. 2004. 